\title{
FEMINISMO Y SOCIALISMO EN LOS ALBORES DEL XX EN COLOMBIA
}

\author{
Angélica Beltrán Pineda \\ Universidad de Antioquia, Colombia \\ antabelp@gmail.com
}

\section{RESUMEN}

El presente artículo aborda la relación problémica entre feminismo y socialismo, tomando como base cuatro experiencias de lucha social desarrolladas en las primeras décadas del siglo xx en Colombia. A partir de estos casos, se exploran las formas de relacionamiento entre las expresiones feministas y socialistas de la época, así como la existencia del feminismo popular en los albores del siglo xx y sus características. Como principales hallazgos se identifican avances y limitaciones en la relación entre feminismo y socialismo en cuanto a lo organizativo y lo programático; a su vez, se verifica la existencia de experiencias feministas liberales y de corriente popular en esta época, con sujetas, reivindicaciones y repertorios característicos, lo cual supone el cuestionamiento del relato histórico de las olas del feminismo. Palabras Clave: feminismo, socialismo, siglo xx, feminismo popular, feminismo socialista.

FEMINISM AND SOCIALISM IN THE BEGINNING

OF THE TWENTIETH CENTURY IN COLOMBIA

\section{Abstract}

This article addresses the problematic relationship between feminism and socialism, based on four experiences of social struggle developed in the first decades of the 20th century in Colombia. From these cases, the forms of relationship between feminist and socialist expressions of the time are explored, as well as the existence of popular feminism at the dawn of the 20th century and its characteristics. As main findings, advances and limitations in the relationship between feminism and socialism in terms of organization and program are identified; in turn, the existence of liberal and popular feminist experiences at this time is verified, with characteristic subjects, demands and repertoires, which supposes the questioning of the historical account of the waves of feminism.

KEYWORDs: feminism, socialism, twentieth Century, popular feminism, socialist feminism. 


\section{INTRODUCCIÓN}

Este ejercicio investigativo busca analizar la relación entre feminismo y socialismo en algunos casos situados en la primera mitad del siglo xx en Colombia, a saber, los procesos liderados por Juana Julia Guzmán en la costa norte, en especial la Sociedad de Obreras Redención de la Mujer (1919); la huelga de mujeres trabajadoras de la Fábrica de Tejidos de Bello desarrollada en 1920 en el departamento de Antioquia, donde resalta el papel de Betsabé Espinal; el Partido Socialista (1919); y el Partido Socialista Revolucionario (1926). Lo anterior se desarrolla a partir del esbozo del contexto histórico del feminismo (enfatizando en su tendencia popular), y del socialismo en las primeras tres décadas del siglo $\mathrm{xx}$, posteriormente se abordan las formas de relacionamiento de la praxis socialista y feminista en los casos señalados.

Los anteriores objetivos de trabajo parten de la identificación de retos en el relato histórico del feminismo, así como de una relación problémica entre el feminismo y el socialismo, dos apuestas emancipadoras. En primer lugar, los retos en el relato histórico del feminismo se generan debido a que la narración histórica del mismo se ha dividido tradicionalmente en «olas» con una periodicidad definida, y unas agendas, por ejemplo, la vindicación del sufragio femenino en los años 50; la sexualidad libre, informada y placentera, en los años 70 . Además, se han identificado repertorios de movilización característicos, como las marchas, publicaciones, desnudos, entre otros; este enfoque lineal de la historia ha sido discutido por autoras como Sandoval (2000) y Thompson (2002), al considerar que lo registrado allí ha sido la historia del feminismo hegemónico, excluyendo multiplicidad de expresiones libertarias existentes en cada época, así como años de lucha, territorios y sujetos que no se incluyen dentro de la delimitación de cada ola, y en los cuales se insinúa que no se adelantaron iniciativas emancipadoras. De acuerdo con Barba, "propongo que aceptemos el vértigo de un pasado desordenado. Y es que si ya aceptamos que hay muchos feminismos, ¿no sería un contrasentido pensar que existe una sola cronología?» (5).

En este sentido, el presente trabajo busca identificar algunas de esas otras prácticas e ideas de mujeres populares que aportaron al proceso feminista, cuyas contribuciones no se dieron en el campo de la producción académica y la discusión política nacional ${ }^{1}$, sino en escenarios propios de las luchas obreras y campesinas, en épocas anteriores a la movilización de mujeres por el sufragio.

En segundo lugar, la relación entre feminismo y socialismo se entiende aquí como la comprensión y desarrollo articulado de la lucha contra el capitalismo, el patriarcado y el colonialismo, concebido como un sistema de dominación múltiple (VALDÉs 78). Actualmente y en el contexto latinoamericano, la real integración de estas luchas feministas y socialistas sigue siendo un reto para las organizaciones políticas (Brenner; Guaramato; SuÁrez). Esto se debe, en primer lugar, a la consi-

\footnotetext{
${ }^{1}$ Escenario en el cual sí incidieron mujeres, principalmente relacionadas con el Partido Liberal, la creación artística y el campo educativo.
} 
deración del feminismo ${ }^{2}$ como algo no prioritario en el marco de la lucha de clases, partiendo de una comprensión de clase social que alberga un reduccionismo económico al dividir las dominaciones de clase que identifica como económicas con las relativas al género y etnia, que ubica de manera errónea como limitadas a la dimensión cultural (ÁLVAREz).

Por otro lado, en algunos sectores socialistas existe la concepción del feminismo como una lucha burguesa y academicista, cuyas reivindicaciones asociadas a los intereses de la clase gobernante podrían solucionarse en el marco del sistema capitalista (APONTE). Además, se ubica en esta discusión la calificación del feminismo como conflictivo, en tanto busca «hilar fino», esto es, identificar las cuestiones cotidianas normalizadas que reproducen estereotipos y ejercicios de dominación; en este sentido, el cuestionamiento de prácticas de las personas que se identifican con el socialismo y que pueden ser incoherentes con los principios de justicia y libertad que lo rigen pone de relieve conflictos como la violencia contra las mujeres al interior de las organizaciones, el desconocimiento del trabajo realizado, entre otras situaciones (FABBRI; SuÁREZ).

Teniendo en cuenta que la relación que aquí se problematiza no se expresa solamente en lo organizativo, es decir, en cómo se articula una organización feminista con una socialista, sino que plantea la cuestión de cómo se complementan y cuestionan mutuamente métodos, presupuestos teóricos y estrategias de transformación social, en el contexto colombiano de principios de siglo, es importante resaltar que las organizaciones feministas de izquierda presentan un reto para integrar el método de análisis de la realidad y las relaciones de opresión propuesto por el marxismo, así como las organizaciones socialistas presentan un reto en la integración de la perspectiva feminista (Mitchell).

Dado lo anterior, se comprende la historia como un escenario de disputa política, cuyos relatos inciden en la construcción de presente y futuro, en tanto herramienta de cuestionamiento y transformación de las relaciones de dominación. De esta manera, en la medida en que se avanza en su lectura crítica se aporta a la cualificación de las organizaciones políticas emancipadoras, de mujeres y feministas.

\section{DECISIONES METODOLÓGICAS Y TEÓRICAS}

Este ejercicio investigativo se realiza en el marco de un paradigma crítico y feminista que implica el reconocimiento de las mujeres como sujetas políticas y de conocimiento; como indica Barbieri, «se trata de producir una teoría o los conocimientos necesarios para liquidar la desigualdad y subordinación de las mujeres: por ello, esta teoría contempla referentes más o menos inmediatos para la acción política feminista» (citada en CASTAÑEDA 14). La investigación feminista no se genera

\footnotetext{
2 Se alude al feminismo en singular ya que en en el marco de este debate socialista se considera en muchas ocasiones una lucha homogénea, desconociendo sus tendencias ideológicas.
} 
por las necesidades de la ciencia en abstracto (CASTAÑEDA 14), sino de la práctica derivada del compromiso social y la necesidad de explicar las condiciones de posibilidad y formas de reproducción de la opresión interseccional; de acuerdo con esto, es importante mencionar que los cuestionamientos que orientan este trabajo no se derivan únicamente de disertaciones teóricas, surgen de las necesidades identificadas en el marco de la militancia feminista y socialista.

Esta investigación tiene un enfoque cualitativo, que en el marco del paradigma crítico se entiende como una forma sistemática y rigurosa de construcción de conocimiento, desarrollada en muestras no muy amplias que permiten la construcción de los datos a través de la profundización en los casos, negando la neutralidad y reconociendo la existencia de intereses al momento de desarrollar una investigación (Colmenares 2012). De acuerdo con esto, para el análisis de los cuatro casos de estudio se empleó la metodología de Investigación Documental ${ }^{3}$, a través de la revisión de archivo del periódico El Luchador y la revista Letras y Encajes. El periódico El Luchador, "Defensor de los derechos del pueblo», fue creado en 1918 en la ciudad de Medellín, ubicada en el departamento de Antioquia. Este periódico de tendencia socialista estuvo relacionado con el directorio departamental del Partido Socialista desde 1919. Por su parte, la revista Letras y Encajes fue fundada en Medellín en 1926 por Teresa Santamaría de González, con la intención de atraer el público femenino a la literatura y el arte, en sus artículos podrían reflejarse elementos ideológicos de tendencia conservadora y liberal.

Se revisaron igualmente documentos personales y fotografías consignadas en el Archivo Fotográfico de la Biblioteca Pública Piloto de Medellín; a su vez, se realizó revisión de literatura, además de entrevistas a personas estudiosas de la época y los casos mencionados como Carlos Uribe y Maria Piedad León. En cuanto a las referencias teóricas, es fundamental mencionar las concepciones de las cuales se parte respecto al feminismo, feminismo popular y socialismo, así como algunas tendencias identificadas en la relación histórica entre el feminismo y socialismo.

Es importante recordar para el abordaje del feminismo y el feminismo popular que las múltiples corrientes del feminismo son una expresión de la diversidad de mujeres que los construyen; el feminismo no puede ser homogéneo cuando partimos de que hay diferenciaciones de etnia, clase social, identidad sexual y de género, edades, territorios, historias. Es así que se asume una definición general de feminismo como punto de partida para las diversas corrientes, de las cuales es aquí el feminismo popular la de principal interés.

En este sentido, retomando elementos de conceptualización de Luna y Fagoaga (1985), el feminismo se entiende como un proceso histórico inacabado de liberación de las mujeres de la dominación interseccional, donde el concepto mujer se comprende a partir de la heterogeneidad social producida por el sistema de dominación múltiple, es decir, más allá de las mujeres blancas y burguesas; asumiendo

\footnotetext{
${ }^{3}$ La investigación documental en este caso es entendida como una estrategia de generación de información basada en el análisis de documentos hemerográficos de carácter histórico (VALLÉs 107).
} 
la inseparabilidad histórica de la condición etnia/clase/género/sexualidad (LuGONES; VIVEROS).

El feminismo popular $r^{4}$ puede entenderse como un proceso emancipatorio de las relaciones de género, clase y etnia, situado en un territorio (cuerpo-espacio), así como en un tiempo determinado. Este es construido por las mujeres populares, es decir, mujeres ubicadas en la subalternidad y cuyo ejercicio político se realiza en relación antagónica con las propuestas de la clase dominante (HALL). «Hablamos de un feminismo popular, insumiso, socialista, que cuestiona a las concepciones patriarcales de ciertas izquierdas, y también a las perspectivas domesticadoras de un feminismo burgués, funcional a las políticas de explotación capitalista» (Korol 5). De ahí que el feminismo popular en muchas ocasiones no se nombre como tal, puesto que su práctica puede no estar rodeada de constructos teóricos, pero sí del reconocimiento de una situación de violencias particulares por el hecho de ser mujeres y la necesidad de trabajar por su transformación.

Por otro lado, se parte aquí de una perspectiva latinoamericana del socialismo, el cual ha tenido múltiples propuestas dada la diversidad social de la región, nombrándolo raizal (FALs, Socialismo), comunitario (GARCÍA), del siglo XXI (СHÁVEz), cristiano o liberal-socializante (URIBE, Los años), entre muchos otros posibles. En este sentido, podría decirse que en general el socialismo es la transición siempre problemática y creativa del capitalismo a otro proyecto de sociedad basado en el bienestar social colectivo y en relación armónica con la naturaleza, sea este el comunismo, buen vivir, comunitarismo, anarquismo, u otros. Como plantea García Linera, «es el campo de batalla entre lo nuevo y lo viejo, entre el capitalismo dominante y el comunitarismo insurgente. Es la vieja economía capitalista aún mayoritaria, gradualmente, asediada por la nueva economía comunitaria naciente» (GARCía 69).

Esta transición se concibe como un proceso promovido tanto por el poder del Estado como por el poder de los sujetos territorializados, lo que implica que la «lucha entre lo viejo y lo nuevo» es un ejercicio cotidiano y se verifica como praxis de las organizaciones sociales y en ocasiones de instituciones estatales, relacionándose entre otras cosas con medidas orientadas a la desprivatización de los bienes comunes, el ejercicio de la democracia paritaria y radical, la producción y propiedad colectiva de los medios de producción, la formación de una ética revolucionaria, entre otras medidas de construcción cotidiana de la apuesta estratégica anticapitalista, decolonial y antipatriarcal.

La relación entre el feminismo y el socialismo ha estado marcada por importantes aportes mutuos; por un lado, se reconoce la contribución generada por autores clásicos del marxismo en el análisis económico de la opresión de las mujeres, así como la desnaturalización de esta dominación basada en argumentos biológi-

4 Cabe aclarar que no se utilizó el concepto de feminismo socialista, por un lado, por la ambigüedad del socialismo en las primeras décadas del siglo xx en Colombia, que complejiza la definición del feminismo socialista en el país, y por otro lado, por considerar que el concepto de feminismo popular es más abarcador, y puede integrar prácticas consideradas o no como socialistas, pero siempre emancipadoras. 
cos e históricos (Álvarez); por otro lado, el feminismo socialista ha revisado críticamente los marxismos, dando relevancia al concepto de reproducción en el análisis del sistema capitalista, lo cual ha derivado en la comprensión interseccional de la explotación, apropiación del trabajo y el cuerpo femenino (FeDERICI), a partir de la investigación de la acumulación originaria, la democracia, la industrialización, entre otros aspectos.

No obstante, pese a los mutuos aportes, diversas investigaciones (ÁLVAREZ; FAbBri; SuÁrez) sustentan la vigencia de la afirmación de Hartmann (2010) respecto a que la "cuestión de la mujer» abordada por el marxismo y los procesos socialistas no ha sido la "cuestión feminista», debido a que la primera se centra en el análisis de la relación de las mujeres con el sistema económico y la segunda aborda también la relación entre hombres y mujeres en el marco del sistema capitalista, lo cual se traduce en que procesos socialistas no siempre se ocupen de integrar el feminismo a su trabajo, sino de incorporar a las mujeres a la causa socialista (ÁlLVAREz), una causa en ocasiones abstracta y donde deben prescindir de muchos de sus intereses y necesidades. Lo anterior es expuesto por Âlvarez al retomar una comunicación de Lenin con Clara Zetkin:

Clara, aún no he acabado de enumerar la lista de vuestras fallas. Me han dicho que en las veladas de lecturas y discusión con las obreras se examinan preferentemente los problemas sexuales y del matrimonio. Como si éste fuera el objetivo de la atención principal en la educación política y en el trabajo educativo. No pude dar crédito a esto cuando llegó a mis oídos. El primer estado de la dictadura proletaria lucha contra los revolucionarios de todo el mundo... i Y mientras tanto comunistas activas examinan los problemas sexuales y la cuestión de las formas de matrimonio en el presente, en el pasado y en el porvenir! (Lenin citado en Álvarez 9).

En la relación feminismo-socialismo se han generado diversas visiones de la cuestión de la mujer, especialmente en lo relacionado con la vinculación de las mujeres como fuerza de trabajo en los diferentes procesos de industrialización, de estas se resaltan aquí cuatro importantes que pueden encontrarse en el contexto colombiano de principios del siglo xx: 1) por un lado, los abordajes que se centran en el estudio de la relación de las mujeres con la producción, y que afirmaron que la vinculación de las mujeres al mercado -saliendo de la esfera privada a la pública- eliminaría la división sexual del trabajo evidente en la asignación del trabajo doméstico a las mujeres; lo anterior implicaría la unidad de mujeres y hombres en su lucha contra el capital, enmarcada en la contradicción obrero/patrón (HARTMANN); 2) por otro lado, se presentaron posturas favorables a la vinculación de las mujeres como fuerza de trabajo, al considerar que el salario ayudaría a reducir la dependencia económica de las mujeres; sin embargo, no se consideraba que esta vinculación garantizara la emancipación para las mujeres (BEBEL); 3) en contraste con lo anterior, principalmente en los primeros momentos de la industrialización, se presentaron posturas al interior de sindicatos socialistas que rechazaban la vinculación laboral de las mujeres debido a que significaba la subversión de los roles de género y restaba opciones de trabajo a los hombres (HarTmanN); 4) finalmente, se encuentran propuestas como las de Federici $(2010,2018)$ y Fraser (2015), que buscan integrar el concepto 
de reproducción a los análisis marxistas y las luchas desarrolladas, reconociendo la apropiación del trabajo femenino como parte de la dominación capitalista, lo cual reforzó la identificación del sustento económico del patriarcado, actualmente funcional al capitalismo.

Como se verá más adelante, estas tendencias en el debate de la cuestión de la mujer y la vinculación de las mujeres como fuerza de trabajo asalariada sirven como guía en la comprensión de la forma de relacionamiento del feminismo y el socialismo establecida en las primeras tres décadas del siglo xx en Colombia en los cuatro casos seleccionados, puesto que la construcción del socialismo en el país no estuvo desligada de las ideas provenientes de algunos países donde estos debates se han generado, como Alemania, Estados Unidos y la URSS.

\section{FEMINISMO Y SOCIALISMO EN LOS ALBORES DEL SIGLO XX EN COLOMBIA}

Las primeras décadas del siglo xx pueden caracterizarse como un periodo de transición de la sociedad colombiana, en el cual diversos aspectos de la vida se modernizan (CORREA). En la época se presentaron procesos de urbanización, industrialización y crecimiento de la economía del país en el marco de la entonces reciente finalización de la guerra de los mil días, todo esto en un contexto internacional de revolución rusa y mexicana, finalización de la segunda guerra mundial y extensión del imperialismo estadounidense en la región latinoamericana (URIBE, Los años). Los anteriores procesos implicaron, entre otras cosas, la migración de mujeres (mestizas, negras e indígenas), niñas y niños del campo colombiano a los poblados urbanos, para proveer de trabajadoras a las nacientes industrias, ya que la fuerza de trabajo masculina estaba menguada por la reciente guerra, además; no se contó con fuerza de trabajo extranjera debido a que la migración internacional fue poca en Colombia (ARCHILA). Es el contexto mencionado, con sus transformaciones y las nuevas contradicciones que presenta, lo que posibilita una alta beligerancia en diferentes sectores de la población, influidos por la construcción de una identidad obrera y campesina que se presentó en la época y que tuvo su ocaso al finalizar la década de 1920, tras múltiples ejercicios de represión, estigmatización y exterminio de las movilizaciones y organizaciones populares, por parte del Estado colombiano (VALVERDE).

En este marco, el feminismo fue una praxis con acogida en el país en las primeras décadas del siglo $\mathrm{xx}$, en la que se presentaron tendencias ideológicas como en otras partes del mundo. Estas tendencias expresaban las situaciones e intereses de las diferentes mujeres colombianas, de ahí que es importante esbozar estas corrientes, sus reivindicaciones y repertorios de acción para enfatizar en las populares, que ocupan este trabajo. De otro lado, en este momento histórico se identifica un importante impulso y desarrollo de las ideas socialistas, que derivan en formas organizativas diversas durante las tres primeras décadas del siglo, y que expresan a su vez diferentes formas de encuentro con el feminismo.

Desde esta perspectiva, en este apartado se aborda un contexto del feminismo en la época, enfatizando en su diversidad ideológica, para concluir presen- 
tando dos experiencias representativas del feminismo popular. Posteriormente, se expone la situación del socialismo en las primeras décadas del siglo $\mathrm{xx}$, haciendo énfasis en la figura de Flores del trabajo, la cual fue creada por el Partido Socialista Revolucionario, y de gran importancia en la relación con los procesos de mujeres feministas señalados.

\subsection{EL FEMINISMO EN LOS INICIOS DEL SIGLO XX Y SUS EXPRESIONES POPULARES}

El feminismo en las primeras décadas del siglo xx existía en Colombia en gran medida como preocupación, este es el punto de partida de la tesis académica de Ricardo Uribe en 1914, cuando afirma que «el feminismo y el socialismo -los dos grandes problemas que conmueven hoy a los países civilizados- surgirán entre nosotros, más o menos tarde, pero surgirán (...) opinamos que el problema feminista es de tanta actualidad en Colombia como en cualquier otro país» (URIBE 4).

Esta presencia del feminismo en Colombia se debió a la influencia de los desarrollos teóricos y políticos estadounidenses, chinos, de Inglaterra y el resto de Europa en el tema; así como en las experiencias latinoamericanas, principalmente argentinas, que fueron llegando al país y generaron opiniones diversas difundidas en gran medida a través de la prensa de finales del siglo XIx. De esta manera, se identifican ideas feministas en Colombia desde 1905, en el marco del Festival Lírico celebrado en el Teatro Bolívar de Medellín, donde María Rojas Tejada -de corriente liberal- presentó una conferencia relacionada con la importancia de la educación para las mujeres, la cual fue valorada por Carlos E. Restrepo 5 como el «primer capítulo de feminismo militante» en Colombia (Reyes 217).

La influencia de las ideas feministas aumentó en las primeras décadas del siglo $\mathrm{xx}$, especialmente en las mujeres populares, debido al contexto de industrialización y la salida de las mujeres del espacio doméstico, lo cual favoreció su socialización y el cuestionamiento colectivo del orden social de la época (Olive; Uribe; VeGa).

Dado lo anterior, cabe afirmar que el feminismo es desde la primera década del siglo xx una lucha polémica y en disputa, que giró alrededor del debate feminismo o feminidad (LuNA), esto implicaba la incomprensión de la propuesta feminista que llegaba al país, desviándose de ser una apuesta emancipadora a una doctrinante en una norma femenina cooptada por parte de sectores como la Iglesia (VILlareal) y partidos políticos tradicionales, lo cual limitaba su carácter transgresor. De esta manera, se presentaron posturas que relacionaban al feminismo con el catolicismo y el cristianismo, así como también existieron perspectivas anarquistas que abogaron por la emancipación femenina, como en la publicación realizada en

5 Carlos E. Restrepo (presidente de Colombia 1910-1914) se relacionó igualmente con el caso de la Fábrica de Tejidos de Bello, que se aborda en ese trabajo, puesto que en 1906 criticó a su gerente propietario, el señor Emilio Restrepo Callejas, por las condiciones laborales a las que sometía a las obreras, lo que Restrepo consideraba que alentaba el «anarquismo» y las huelgas (citado en VEGA 135). 
1925 por Ana María García invitando a las mujeres a dejar de ser el instrumento de los hombres, llamando al rechazo de la ignorancia y a construir la revolución social (GARCía citada en Vega).

Es importante señalar que el feminismo tuvo impactos y adoptó estrategias diferenciadas según la clase socioeconómica de las mujeres que lo acogieron. Si bien fue apropiado por las mujeres de clase media-alta, en su mayoría cercanas al Partido Liberal, también llegaron sus ideas a las mujeres obreras, quienes promovieron huelgas, centros de estudio y publicaciones feministas (Zapata, Semanalmente). De igual manera, las reivindicaciones que unas y otras generaban eran diversas, de modo que las principales demandas de las mujeres liberales en la época fueron alrededor de la posibilidad de disponer de sus bienes materiales en el marco del matrimonio -capitulaciones matrimoniales-, el acceso a la educación y el derecho al voto. Por su parte, en las mujeres trabajadoras se presentaron reivindicaciones relacionadas con la mejora de sus condiciones laborales, ${ }^{6}$ apoyo a campañas feministas (FALs 148B) y la generación de procesos autónomos de educación. Lo anterior no pretende sugerir que ambos tipos de reivindicaciones no fuesen complementarias y aportantes a los derechos humanos de las mujeres; no obstante, al partir de las realidades concretas de las mujeres se priorizaban unos u otros asuntos.

La anterior diversidad del feminismo según la clase social señalada se refleja en el Congreso Internacional Femenino realizado en 1930 en la ciudad de Bogotá, que contribuyó a la consolidación del feminismo en el país. Allí pudo verse el encuentro de personalidades femeninas en su mayoría nacionales y algunas internacionales, las primeras fueron delegadas por los gobernadores de 14 departamentos para la asistencia al evento (COHEN) y las segundas relacionadas con los demás congresos internacionales realizados anteriormente, mujeres referentes por las luchas desarrolladas en sus países.

En este Congreso Internacional se abordaron las problemáticas relacionadas con el trabajo, frente a las cuales se proponía una campaña para la sindicalización de las mujeres trabajadoras, que se veían sometidas a sueldos irrisorios y situaciones vulnerantes; de esta manera, en el marco del evento María Eastman manifestó preocupación por las mujeres de clase media señalando que «ellas son nuestras hermanas, están sufriendo, y es necesario ayudarlas» (citada en CoHen 83). Esto permite afirmar en primer lugar que estas mujeres trabajadoras no estuvieron presentes en el Congreso, ya que se habló por ellas y no desde ellas, y por otro lado, que las asistentes a este congreso trabajaron alrededor de una agenda propia, representativa de sus intereses y realidades, aunque no desarticulada de las personas empobrecidas del país.

${ }^{6}$ Para Ricardo Uribe (1914), la lucha por mejores condiciones laborales para las mujeres en las primeras décadas del siglo xx es una reivindicación feminista, en tanto se da en un contexto en el cual las mujeres trabajadoras eran estigmatizadas por abandonar su lugar natural en el mundo: el hogar, por lo que luchar por esto era abogar por la independencia económica de las mujeres, primer factor de dominación social, según el autor. 
Por su parte, en la revista Letras y Encajes se abordó la necesidad «urgente que tiene Colombia de una "élite" femenina sólidamente preparada para la acción en el campo social» ("Instituto de Cultura Femenina», 569), refiriéndose al tema educativo; esta visión soporta la construcción de las luchas de las mujeres y la garantía de sus derechos de manera excluyente, puesto que en la élite propuesta no tenían cabida las mujeres populares (empobrecidas, mestizas, indígenas y negras).

Este escenario del feminismo en la época demuestra dos cuestiones de importancia, en primer lugar, que hablar de este proceso emancipatorio en las primeras décadas del siglo xx en Colombia no es anacrónico (ya que el imaginario de la existencia del feminismo a partir de 1940 se encontró de manera recurrente en el desarrollo de este trabajo), y por otro lado, que el feminismo no fue sólo dirigido o creado por mujeres clase media-alta; por el contrario, fue promovido por mujeres populares cuyas condiciones de vida posibilitaron cuestionamientos respecto a su condición de mujeres trabajadoras que habitaban nuevos espacios, eran precarizadas y violentadas. Es como parte de estas experiencias feministas desarrolladas por mujeres populares que en este trabajo se reivindica a la Sociedad de Obreras Redención de la Mujer y la huelga de trabajadoras de la Fábrica de Tejidos de Bello de 1920, dado que representan una interesante confluencia de feminismo y socialismo en la época.

\subsection{La Sociedad de Obreras Redención de la Mujer}

El Centro de Emancipación Femenina y la Sociedad de Obreras Redención de la Mujer fueron creados en Montería en 1916 y 1919 respectivamente, por iniciativa de Juana Julia Guzmán, en el marco de las luchas desarrolladas con su pareja, el anarquista italiano Vicente Adamo (ELJACH). Juana Julia nació en Corozal Sucre en 1892 y falleció en 1975 a sus 85 años, desarrolló múltiples luchas en la época en el marco de las organizaciones de mujeres mencionadas, así como en la Sociedad de Obreros y Artesanos de Córdoba y el Baluarte Rojo de Lomagrande, fundados en 1918, en compañía de las mujeres que acogieron su llamado «mujeres bailadoras de fandango, lavanderas, fritangueras, cocineras como Pacha Ferias, Agustina Medrano, Antonia Espitia, Marcelina Agamez, Mercedes Vidal y Josefina González» (Bonilla 56). La lucha desarrollada en el marco de la Sociedad de Obreras fue entre otras cosas por condiciones de trabajo dignas y educación, como relató Juana Julia:

Queríamos redimirnos nosotras mismas, porque esas mujeres eran muy martirizadas. De coger las blancas a las pobres sirvientas y darles calderetazos y tirarles leche caliente encima. Nos organizamos con reuniones los martes y sábados para que siquiera aprendieran a defenderse, que no se dejaran y que no había prisión por deudas (citado en Fals 143A).

7 Si bien el Centro de Emancipación Femenina contiene también una importante relación entre feminismo y socialismo para la época, se da prioridad a la Sociedad de Obreras Redención de la Mujer debido a las posibilidades de acceso a la información. 
Aquí se identifica el componente interseccional que caracteriza al feminismo popular, específicamente cuando Juana Julia hace referencia a la relación de las jefas blancas con sus trabajadoras, a quienes maltrataban, resaltando el componente de género, clase y etnia que marcó a la problemática y a la Sociedad de Obreras.

El proceso revolucionario liderado por Juana Julia Guzmán y Vicente Adamo adoptó como estrategia la construcción de organizaciones gremiales -entre estas la Sociedad de Obreras Redención de la Mujer-, y la creación de organizaciones de carácter electoral separadas de las anteriores para "evitar la potiquería» (FALS 150B). De tal manera que las obras realizadas por estas sociedades, como el centro obrero, un hospital y una biblioteca (FALs 2002), eran iniciativas realizadas conjuntamente en las organizaciones mixtas, aunque considerando las necesidades educativas de las mujeres.

Respecto a Juana Julia, cabe recordar que esta mujer fue una de las tantas que migraron a inicios de siglo del campo a los poblados urbanos. Trabajó como clasificadora de hojas de tabaco hasta 1916, cuando llegó a Montería a emplearse como trabajadora doméstica, cantinera y ventera. Pese a que Juana Julia no pudo acceder a la escuela, su liderazgo fue fundamental para el desarrollo de procesos revolucionarios en la costa norte y llegó a tener tal importancia que Vladimir Llich Ulianov (Lenin) le envió una carta exaltando su labor, la cual fue encontrada por Orlando Fals Borda en sus investigaciones para la Historia doble de la costa (ELJACH).

Juana sería conocida más tarde como «la robatierra» por las luchas de recuperación de tierras libradas con los campesinos, especialmente en Lomagrande. Fue enviada a la cárcel, estigmatizada, perseguida y violentada por su ejercicio político, aun así se mantuvo como presidenta de la Sociedad de Obreras Redención de la Mujer, y segunda gerente de lo que más tarde sería el baluarte Nueva Galicia (espacios territoriales recuperados por los campesinos para ser habitados y realizar trabajos comunales). Su lucha no concluyó en las primeras décadas del siglo xx, puesto que después del descenso de los proyectos realizados en la época, retomó su militancia con la Asociación Nacional de Usuarios Campesinos (ANUC) en 1970, fortaleciendo el comité de mujeres de dicha organización (FALS).

\subsection{La huelga de trabajadoras de la Fábrica de Tejidos de Bello}

Como otro caso de importancia de la época resalta la huelga de trabajadoras de la Fábrica de Tejidos de Bello, debido a que esta experiencia contribuyó al proceso de emancipación femenina desde una perspectiva feminista y socialista. La huelga inició el día 16 de febrero de 1920 por iniciativa de Adelina González, Teresa Tamayo y soporte activo de Betsabé Espinal, Trina Tamayo, Teresa Piedrahita, Matilde Montoya, Carmen Agudelo, Rosalina Araque y Bedalina del Valle (Detective). Es importante mencionar que este no había sido el primer intento de huelga en la fábrica, puesto que anteriormente se había generado la iniciativa, pero todas las trabajadoras relacionadas fueron despedidas (El Correo Liberal, citado en Martínez y Uribe 129). 
La huelga se desarrolló en un ambiente de amplia acogida mediática y social, hasta el punto que el alcalde de Medellín y la policía parecieron apoyarlas. El apoyo de diversos sectores de la población se expresó a través de la realización de ollas comunitarias que contaron con el aporte de donantes del territorio del Valle de Aburrá y de los vecinos de la fábrica, así como con expresiones artísticas y discursos políticos que alentaron a las huelguistas y permitieron concluir la huelga exitosamente. Así, el día de culminación oficial de la huelga se había logrado todo lo planteado en el pliego de peticiones; según los acuerdos realizados con la empresa se estableció:

1. ${ }^{\circ}$ Horas de trabajo: 9 horas 50 minutos, las cuales se repartirán de acuerdo con las Obreras, para las salidas a desayunar, almorzar y tomar el refrigerio entre el almuerzo y la comida, para que de este modo queden constituidas ocho horas diarias de trabajo. 2. ${ }^{\circ}$ Salarios: la Compañía garantiza a las obreras un aumento del cuarenta por ciento; pero se reserva el derecho de repartir ese aumento de una manera equitativa, con el objeto de evitar que queden unas más favorecidas que otras. $3 .^{\circ}$ Libertad de calzarse. Las obreras pueden hacer de esta materia lo que les plazca y lo que les acomode. $4 .^{\circ}$ Cambio de Caciques: la Compañía garantíza a las Obreras su derecho justo y moral de presentar cargos concretos contra los tres Caciques, cargos que serán atendidos y estudiados íntimamente por el Gerente, por la Junta Directiva y, naturalmente por las autoridades del Distrito (Alcalde o Juez Municipal). La compañía reservará imparcialidad y de acuerdo con la justicia despedirá a los empleados que resulten culpables («Solución de la huelga»).

Lo anterior fue un triunfo de gran magnitud y un mensaje fuerte para la época sobre la beligerancia de las mujeres, solidaridad de la comunidad, medios de comunicación y organizaciones, así como de posibilidades de éxito en las reivindicaciones de las personas trabajadoras. Así mismo, es relevante el hecho de dársele trámite público y solución al objetivo principal de la huelga, que era el abuso sexual al cual estaban sometidas, tal como lo afirmó Betsabé:

El objeto primordial del actual movimiento, es principalmente, los abusos y arbitrariedades de los tres caciques Jesús Monsalve (Taguaica), Teódulo Velásquez, y Manuel J. Velásquez; estos señores son los verdaderos responsables y sobre los cuales se deben recargar todas las pérdidas que ha proporcionado este conflicto (Detective).

En esta declaración puede identificarse que el cuestionamiento de las relaciones de género fundamentó la movilización, puesto que, además de romper la normalización de las violencias sexuales que sufrían, cuestionaron estereotipos:

Otra cosa que me llama la atención, es que es digno de marcada burla, es que hay hombres tan bobos, que creen que las mujeres somos muy flojas, no hay tal, con menos fuerza, eso sí, pero con más fuerza de voluntad (citada en Detective).

En relación con lo anterior, en la época llamó mucho la atención la reticencia de los hombres que trabajaban en la fábrica a salir a la huelga, por lo que fueron objeto de burla por parte de las mujeres huelguistas, utilizando argumentos contra 
su virilidad. Otra transgresión de importancia fue la negación de las huelguistas a ceder a las recomendaciones de cese de la huelga brindadas por el cura del municipio de Bello, así como no atender a la visita del obispo al municipio (Tintorero), siendo esto muy polémico en un municipio de Antioquia en 1920, donde la Iglesia tenía gran control sobre la vida social y política.

Como puede notarse, si bien muchas mujeres hicieron posible el desarrollo de esta huelga, resaltó el rol de Betsabé Espinal, quien fue nombrada a sus 23 años como delegada de las obreras a una Asamblea Socialista realizada en el marco de la huelga, fue muy reconocida por su oratoria y capacidad de conducción de las trabajadoras durante la movilización, aportando a su desarrollo exitoso. De esta manera, Betsabé narraba: «La policía que custodiaba los salones de la fábrica y que presenciaba todo lo ocurrido, al terminar mi palabra arrojaron los rifles sobre el suelo, para poder aplaudirme y dar de este modo una muestra de su civilización y de su entusiasmo» (citada en Detective).

Betsabé Espinal nació en 1896 y murió en 1932 en el municipio de Bello, hasta el momento se conoce poco sobre su historia; sin embargo, se sabe que su madre estuvo recluida en el hospital mental de Bello, que tenía hermanos y que para el momento de su muerte -a causa de una electrocución accidental- era jefa del taller textil en el patronato de obreras de la Fábrica (URIBE).

\subsection{El APORTE DE LAS MUJERES POPULARES AL FEMINISMO EN LOS ALBORES DEL SIGLO XX}

La Sociedad de Obreras Redención de la Mujer y la huelga de trabajadoras de la Fábrica de Tejidos de Bello en 1920, así como las dos mujeres que representan estas luchas hasta la actualidad, fueron una valiosa contribución al proceso histórico de emancipación de las mujeres. Uno de estos aportes fue la transgresión de los imaginarios de feminidad establecidos; en el caso de Juana Julia, sus adversarios cuestionaban la masculinidad de la gran cantidad de hombres que la seguían, de manera que fue en este contexto donde ella acuñó la frase «el cobarde no hace historia» (FALS 144A).

Por otro lado, en el caso de la huelga en Bello, fue clara la admiración por la valentía de las huelguistas, como se publicó en El Luchador: «Esta vez queda demostrado que la mujer sí sirve para algo más que para arreglar la casa y criar los hijos. Es un espectáculo digno de verse y meditarse esas obreras rebeldes que no quieren continuar bajo el yugo del amo explotador» (Zapata, Obreras).

Este cuestionamiento de los imaginarios derivó en el «elogio» que recibieron tanto Juana Julia como Betsabé en la prensa obrera por su «virilidad» (ELJACH; LuIs), puesto que la actuación de estas mujeres no se correspondía con los imaginarios hegemónicos de la feminidad -asociados a aspectos poco valiosos- y sí con los de la masculinidad -mejor valorados socialmente-. Además del impacto en el imaginario femenino tradicional, lograron hablar del machismo, la sexualidad, los derechos para las mujeres trabajadoras, y tuvieron éxito en sus luchas. 


\section{EL SOCIALISMO Y LA FIGURA DE FLORES DEL TRABAJO}

Es importante mencionar que se hace alusión a un socialismo colombiano de inicios del siglo $\mathrm{xx}$, derivado de corrientes radicales del liberalismo de finales del siglo XIX (CORREA), y que recibe influencia de los procesos revolucionarios que venían en desarrollo en América Latina y Rusia. Este socialismo recoge elementos del anarquismo, la corriente bolchevique, la masonería, el radicalismo liberal, el socialismo cristiano y el espiritismo (NúŃEZ citada en FLórez).

Los partidos políticos socialistas creados en las primeras décadas del siglo fueron en 1916 el Partido Obrero; en 1919 el Partido Socialista; en 1926 el Partido Socialista Revolucionario (PSR); y en 1930 el Partido Comunista. En este transcurrir se presentaron transformaciones en dichos partidos políticos en cuanto a sus configuraciones ideológicas, reivindicaciones, estrategias, métodos de trabajo y difusión de las ideas (CARO). De este modo, durante estas décadas se adoptaron posturas reformistas y revolucionarias, se potenciaron iniciativas de prensa obrera, se estructuraron organizaciones nacionales, entre otros aspectos.

El nacimiento del Partido Obrero se basó en organizaciones creadas desde 1910 que buscaron ocuparse de las necesidades de esta población, algunas fueron la Unión de Industriales Obreros (1910) y la Unión Obrera de Colombia (1913) (CAro, 2017). Por su parte, en el Partido Socialista (1919) la estructura organizativa creada le permitió vincularse con luchas como las de las mujeres; así, se generó una forma de trabajo basada en la creación de directorios municipales, departamentales y uno nacional (CARO), que debían organizar los diferentes gremios y acompañar las luchas que se generaban en las regiones, lo cual fue efectivo en el caso trabajado en el apartado anterior con respecto a la huelga de Bello en 1920.

Por su parte, en el caso del PSR destaca la importancia de las giras realizadas para la organización de diferentes gremios en el territorio nacional, aquí es importante mencionar la figura de Flores del trabajo, una suerte de reinado que elegía mediante concursos a las mujeres más carismáticas y representativas de las luchas obreras. Para su elección, cada persona votaba y el valor del voto era de 10 centavos, esto permitía la recolección de dinero para la causa socialista (VILlareaL). Las Flores del trabajo incidieron positivamente en la participación de las mujeres en espacios políticos visibles, como fue el caso emblemático de María Cano, quien resaltó por su gran capacidad de oratoria, transgrediendo estereotipos femeninos tradicionales de la época ${ }^{8}$ (VILla).

Uno de estos estereotipos cuestionados fue el de las mujeres confinadas a los espacios privados y desinteresadas en la politica, ya que María Cano se caracterizó por habitar e incidir espacios públicos con un rol de liderazgo.

${ }^{8}$ Uno de los estereotipos que cuestionó fue la maternidad como propósito de la vida femenina. Frente a su decisión de no ser madre, María Cano afirmó en un discurso en Popayán: «Soy mujer y en mi entrańa tiembla el dolor al pensar un hijo que pudiera ser un esclavo» (citada en Toвón 106). 
Usted acusa de conspiradores a mis amigos del PSR y me quiere excluir a mí de tal responsabilidad porque supuestamente estoy llevada y convencida por ellos, o sea, no me otorga la posibilidad de criterio personal. En este país donde la mujer habla es a través del cura, del marido o del padre, hay esa costumbre. Pero ese debate no se lo voy a hacer. La gente sabe quién soy y cuál es mi criterio (citada en Correa 13).

El anterior es un fragmento de una carta enviada por María Cano al secretario general del Partido Comunista, que permite identificar el rol pasivo asignado a las mujeres en la época, incluso desde el seno del comunismo.

\section{FORMAS DE ENCUENTRO DEL FEMINISMO Y EL SOCIALISMO}

Como se mencionó anteriormente, hablar de feminismo y socialismo implica principalmente referirse a cómo se complementan o se diferencian sus métodos y teorías, lo cual implica trascender las relaciones organizativas. De esta manera, cabe señalar que en Colombia la postura de personas y organizaciones socialistas frente al feminismo, las violencias contra las mujeres y roles de género derivados del patriarcado fue heterogénea y en muchas ocasiones contradictoria; no obstante, se establecieron relaciones orgánicas, programáticas y estratégicas.

En el siguiente apartado se abordan algunas formas de encuentro y desencuentro de experiencias feministas y socialistas como la Sociedad de Obreras Redención de la Mujer, la huelga de trabajadoras de la Fábrica de Tejidos de Bello en 1920, el Partido Socialista y el PSR; lo anterior se dará a partir de la identificación de algunas visiones que aportaron al debate sobre la cuestión de la mujer y el feminismo, la relación programática entre feminismo y socialismo que se devela en los casos mencionados y, finalmente, la relación orgánica que se estableció.

\subsection{Debates SObre la CUESTiÓN DE LA MUJER Y el Feminismo}

La prensa obrera, en especial los órganos de difusión de los partidos políticos en diferentes regiones del país, permite identificar algunas tendencias en la concepción del feminismo y el rol de las mujeres en el socialismo. Muestra de ello son las publicaciones de El Luchador en el departamento de Antioquia, donde se hacía seguimiento a las condiciones laborales de las mujeres, sea cuestionando el hecho de que trabajaran o exigiendo condiciones de trabajo dignas para ellas (АтеноRTÚA). A su vez, se publicaban episodios de violencias contra las mujeres exigiendo justicia a las autoridades competentes ("La desgracia», 1919), y se comentaba acerca del rol de las mujeres en la sociedad, la política y la revolución.

En este trabajo se identifica la existencia de posturas contradictorias en los partidos sobre la temática en lecturas de la realidad y propuestas conservadoras desde el socialismo sobre las mujeres y el feminismo. De esta manera, en El Luchador-como órgano regional del Partido Socialista-, por un lado, se encuentran planteamientos conservadores, donde el esencialismo hacia la mujer considerada como 
madre y reina del hogar imperaba (NúNÉEZ 184), mientras que, por otro lado, se evidencian mensajes a favor de la emancipación femenina y de su participación política. En este sentido, en 1918 el periódico socialista El Luchador saca una nota de prensa con la pregunta «¿Cuándo es más fea la mujer?»:

Es fea la mujer cuando habla demasiado. Más fea cuando ríe por ostentación. Mucho más fea cuando en el templo vuelve el rostro atrás para mirar lo que pasa. Peor, cuando se ocupa en asuntos de política. Feísima, cuando no saluda. Atroz, cuando se ocupa en hablar mal de los demás [...]. Terremoto, cuando descuida sus quehaceres domésticos, para cuidar, como un ídolo, su belleza siempre efímera, sin acordarse de que la vida es un sueño («¿Cuándo es más fea la mujer?», 1918).

Puede notarse que en este fragmento se tiende a valorar a las mujeres a partir de su estética y su comportamiento, a generar una norma para las mujeres madres, casadas y obreras desde principios conservadores y misóginos, que inhiben la participación política de las mujeres al relegarlas al espacio doméstico a partir de la naturalización de este trabajo. Este tipo de opiniones se encontraron de manera recurrente en el periódico.

Otra postura interesante hallada en El Luchador es la que relaciona la participación de las mujeres en la revolución con concebir y educar hijos obreros como militantes. Lo anterior refleja la reproducción de valores conservadores sobre la feminidad, la cual para el caso está al servicio del socialismo. Desde esta perspectiva, no se considera a las mujeres como sujetas activas en el marco de la revolución, pues su rol se basa en el acompańamiento a los hombres, sea como madres o esposas, para que estos se desarrollen como sujetos políticos.

Pese a lo anterior, también se identifican posturas favorables a la participación política de las mujeres. En algunos escritos El Luchador exalta iniciativas de emancipación femenina, y propone interpretarlas como parte del «progreso de la humanidad» (ZAPATA), en este sentido se publican noticias sobre Antioquia y Montería:

Con el título de «Feminismo» va a aparecer una revista dirigida por señoritas, entre las cuales se cuenta la intelectual señorita Romelia Gómez. En Montería, según se nos comunicó, fundaron un centro feminista obrero, que tiene ya biblioteca popular y salón de lectura, y piensa dar a la publicidad un órgano que defienda los intereses de las obreras y damas de esa región (ZAPATA).

Tanto la revista Feminismo como el Centro Feminista Obrero son iniciativas que demuestran la acogida del feminismo por parte de mujeres populares, relacionadas con las ideas socialistas de la época. A su vez, demuestran el interés del Partido Socialista por su ejercicio de organización.

Lo anterior permite afirmar que aunque se estuviera en la empresa de construir el Partido Socialista, que como se verá más adelante tenía algunas ideas definidas sobre la situación de las mujeres, no había cohesión en las ideas que se promocionaban en su órgano regional respecto a esta temática; por el contrario, abundaron las contradicciones entre tendencias favorables y desfavorables para los derechos de las mujeres y su consideración como sujetas políticas. A pesar de todo esto, el femi- 
nismo y la cuestión de la mujer fue una problemática que se abordó en el periódico, lo cual pudo contribuir al debate y a la difusión de ideas favorables a las luchas de las mujeres.

\subsection{RELACIÓN PROGRAMÁTICA ENTRE EXPERIENCIAS FEMINISTAS Y SOCIALISTAS}

Además del debate expresado en la prensa obrera frente a la lucha feminista y las mujeres, que da cuenta de una relación no siempre favorable entre ambas luchas, en el campo programático y reivindicativo de las organizaciones estudiadas pueden identificarse igualmente puntos de encuentro.

En el pliego de peticiones de la huelga de trabajadoras de la Fábrica de Tejidos de Bello en 1920 se identifica articulación de reivindicaciones feministas y socialistas, donde se conjugó la exigencia del cese del abuso sexual hacia las trabajadoras de la fábrica con la demanda histórica de los tres ochos (ocho horas para dormir, ocho horas para trabajar, ocho horas para educarse) y el aumento del salario, entre otros puntos del pliego. Si bien las obreras en huelga no llegaron a nombrar ni al socialismo ni al feminismo, la lucha que adelantaron se inscribió en los objetivos de ambas praxis al confrontar las contradicciones clásicas obrera/patrón y las propias del género (mujer/hombre).

En el marco de la Sociedad de Obreras Redención de la Mujer se identifica que los procesos desarrollados en la costa norte colombiana se rigieron igualmente por un socialismo moderado en el marco del Partido Socialista en construcción (FALS 147B). En este caso, algunas de sus aspiraciones fueron el «estímulo a la unión y solidaridad de los trabajadores como clase proletaria universal y (...) apoyo a campañas feministas, antialcohólicas y de democratización del trato personal ("camarada" y "compañero")» (FALS 148B), lo que explícitamente seńalaba la confluencia de feminismo y socialismo en la Sociedad de Obreras y demás organizaciones relacionadas de la costa norte.

Por su parte, el Partido Socialista en 1919 acoge la Plataforma Socialista propuesta por la Confederación Obrera de Bogotá, donde se indica que el socialismo debe propender,

por cuantos medios estén a su alcance, porque la mujer tenga las mayores garantías posibles en guarda de su persona e intereses; porque se la proteja contra la dilapidación del marido vicioso o del detentador de sus bienes; porque su trabajo sea remunerado, según sus aptitudes, lo mismo que el del hombre; porque se la asegure en la percepción de su salario en todo caso comprobado de enfermedad, y especialmente durante los 30 días antes y después de la maternidad; porque los atropellos a su honestidad y decoro sean sancionados severamente por medio de leyes que establezcan la probanza y el castigo de modo eficaz, y porque no se la obligue a trabajar por ningún motivo antes de los 12 años y después de los 60 (Confederación Obrera de Bogotá, 1919).

Estos objetivos demuestran preocupación desde el Partido Socialista por las problemáticas de las mujeres acogiendo reivindicaciones relacionadas con la mejora 
de condiciones de trabajo para ellas y rechazo al trabajo infantil, paridad laboral, licencia de maternidad y justicia ante las violencias de género. Cabe resaltar que no se abordaron otras reivindicaciones importantes de la época como la educación y la participación política a través de sindicatos u otros escenarios.

En conclusión, puede notarse la confluencia de propuestas y reivindicaciones feministas y socialistas en las experiencias abordadas. En el caso de la Sociedad de Obreras en Montería la relación fue explícita, mientras en el caso de la huelga en Bello en 1920 y en los partidos políticos se encuentra de manera implícita, no nombrada. Pese a lo anterior, todas las experiencias estudiadas demostraron haber recibido influencias de estas ideas emancipadoras y aportaron a la consecución de derechos para las mujeres trabajadoras desde una perspectiva revolucionaria.

\subsection{RELACión ORGÁNiCA}

En cuanto a la relación orgánica entre feminismo y socialismo, deben señalarse inicialmente los espacios de coordinación generados entre el Partido Socialista y las obreras de la fábrica para la realización de la huelga de Bello en 1920; por otro lado, cabe mencionar las iniciativas de las organizaciones de la costa norte para la construcción del Partido Socialista nacional y, finalmente, se abordará la figura de Flores del trabajo como forma de encuentro del PSR con algunas luchas de las mujeres, especialmente en Bogotá y Antioquia. Estas experiencias demuestran el encuentro organizado de iniciativas y organizaciones con adscripción a las luchas socialistas y feministas en las primeras décadas del siglo.

En primer lugar, en la huelga de trabajadoras de la Fábrica de Tejidos de Bello de 1920 puede encontrarse evidencia del acompañamiento que realizaron personas integrantes del Partido Socialista a la preparación de la huelga y a su sostenimiento (Martínez y Uribe) desde 1916 con el registro de la presencia de Benedicto Uribe ${ }^{9}$ azuzando a las obreras de esta fábrica para que se manifestaran por sus condiciones de trabajo (URIBE). Lo anterior permite negar el relato que caracteriza a esta huelga como espontánea, y por otro lado, aporta datos sobre las formas de articulación de las organizaciones socialistas con los procesos de lucha de mujeres y feministas.

De acuerdo con esto, la articulación del Partido Socialista con esta huelga se estableció mediante un acompañamiento directo a través de diferentes mecanismos; por un lado, desde la generación de conferencias (URIBE, Telegrama), lo cual tuvo un impacto notorio en la cualificación del discurso de las lideresas, por ejemplo el de Betsabé Espinal; a través de la recolección de dinero para el sostenimiento de la huelga («jobreras! ¡obreros!»,1920); además, se brindó acompañamiento para la orientación de la movilización hasta su culminación oficial a través de reuniones de personas delegadas del Directorio Departamental y las obreras («La huelga

\footnotetext{
9 Uribe fue militante del Partido Socialista en Medellín pocos años después de este episodio, allí sostuvo su apoyo a la huelga de trabajadoras de la Fábrica de Tejidos de Bello generada en 1920.
} 
de Bello», 1920); se generaron acciones de movilización que ayudaron a presionar a los dueños de la fábrica (Herrera, 1920); y, finalmente, existió un importante compromiso con la difusión mediática de la huelga, lo cual aportó a la amplia acogida y legitimidad que tuvo la huelga para la población antioqueńa, al punto de ser apoyada por el alcalde de Medellín (Detective).

Además de lo anterior, se desarrolló una Asamblea Socialista, donde Betsabé Espinal, Matilde Montoya, Trinidad Tamayo y Teresa Piedrahita fueron elegidas como representantes de las obreras ("La huelga de Bello», 1920), demostrando una relación formal entre las obreras y el Partido Socialista. Finalmente, es importante mencionar que esta huelga fue altamente valorada por el Directorio Socialista nacional y departamental, de tal manera que fue estimada como un «triunfo del socialismo en Antioquia» ("Triunfo del socialismo en Antioquia», 1920). De este modo, el 20 de febrero el Directorio Nacional envió una carta a Betsabé exaltando su trabajo.

El Directorio Ejecutivo Nacional Socialista le envía a la señorita Betsabé Espinal un efusivo, cordial y sincero voto de aplauso por su digna actitud, asumida en la huelga de las obreras de la Fábrica de Tejidos de «Bello» de Medellín, y a la vez la excita a que continúe la gloriosa tarea de redimir a sus compañeras de labor (Directorio Socialista Nacional, 1920).

En segundo lugar, se evidencia que la lucha liderada por Juana Julia Guzmán y Adamo estuvo articulada a otras organizaciones e iniciativas nacionales. Uno de los objetivos de esta articulación fue la creación de un primer Partido Socialista en el marco de la Asamblea Obrera de la Costa Atlántica, realizada en Montería en enero de 1921. A su vez, este proceso estuvo relacionado posteriormente con María Cano, Raúl Mahecha y Torres Giraldo entre otros socialistas reconocidos nacionalmente, los cuales abogaron por la nacionalización de Adamo en el momento en que se le expulsó del país en el marco del Gobierno de Miguel Abadía Méndez (FALs).

Finalmente, las Flores del trabajo bien pueden ser leídas como una reproducción del estereotipo femenino donde las mujeres son utilizadas como objetos decorativos, lo cual no se discutirá aquí. A pesar de esto, esta figura permitió el acercamiento de organizaciones y luchas de las mujeres al PSR, así como la posibilidad de algunas mujeres para sobresalir en la política nacional de tendencia revolucionaria. Tal es el caso de María Cano, la Flor del trabajo de Antioquia, y de Sofía López, Flor del trabajo de Bogotá, quienes acompañaron y agitaron movilizaciones de mujeres en la época,

En algunos de los discursos de las mujeres escogidas como Flor de Trabajo se notaba la manera como eran recepcionadas algunas de las reivindicaciones más importantes de las trabajadoras. Por ejemplo, en 1926 Sofía López en su mensaje a los obreros anunciaba que se proponía: «2. Trabajar porque los patronos reconozcan la justicia del principio de que a igual trabajo se reconozca igual salario, sin distinción de sexos ni edades [...] 3. Declarar por todos los medios... guerra sin cuartel al analfabetismo femenino", dos reivindicaciones que hacían parte de la lucha mundial de las mujeres, tal y como se agitaban en ese momento en diversos países de Europa y América Latina (López citada en VeGA 153). 
Dicha relación de las Flores del trabajo con las luchas de las mujeres, además de confirmar la relación de las luchas desarrolladas en Colombia con otros países del continente y el mundo, mostraba una forma concreta en la cual se promovió el debate y se posicionaron puntos específicos desde las mujeres en el PSR. Las Flores del trabajo fueron de gran visibilidad pública, pero no llegaron a ocupar escenarios de dirección y María Cano, una vez que se diluyó el PSR y se conformó el Partido Comunista, fue excluida de este último por ser mujer, es así que el secretario general de este partido afirmó: «El pueblo antioqueño es antimatriarcal y la presencia de una mujer entre los obreros los asusta, los espanta, los obreros no quieren tener cuentas con mujeres» (citado en Acosta 69).

La relación orgánica abordada da cuenta de las importantes relaciones entre organizaciones feministas y socialistas, que fueron conflictivas y diversas de acuerdo al partido político (PS, PSR, PC). La articulación fue heterogénea dado que las experiencias abordadas se dan en años y territorios diversos, lo que las sitúa en contextos municipales más favorables o adversos para la recepción de discursos revolucionarios. Así, fue favorable la influencia de ideas provenientes de otros países en las luchas desarrolladas en la costa norte, mientras fue adverso para la acogida de las ideas feministas el marcado poder de la Iglesia sobre la vida antioqueña, en cuyo marco se desenvuelve la huelga en 1920.

Además, podría decirse que si bien existió un avance en el reconocimiento de algunas necesidades de las mujeres en el marco del Partido Socialista y posteriormente en el PSR, relacionadas con la dignificación de las condiciones laborales y la justicia ante situaciones de violencias de género, entre otros asuntos, se presentaron limitaciones en la integración del feminismo con estas luchas. De esta manera, en los casos abordados el feminismo no se nombró por parte de las organizaciones socialistas nacionales, ni se reivindicó como parte integradora del socialismo, pese a que para el momento en Colombia y otros países del mundo (como Alemania y la URSS) existían desarrollos del feminismo socialista entre las mujeres obreras. Esta exclusión del feminismo de la praxis socialista se profundizó cuando el PSR se extinguió y surgió el Partido Comunista, con una influencia soviética en la cual esta lucha no tuvo cabida.

\section{CONCLUSIONES}

El feminismo era una propuesta política en disputa en las primeras décadas del siglo $\mathrm{xx}$, como lo sigue siendo hoy. En este camino las ideas socialistas jugaron un importante rol en la potenciación de la participación política de las mujeres y la construcción de sus propias organizaciones e iniciativas transformadoras, tales como huelgas, publicaciones, entre otras. De ahí que es importante recordar que el feminismo como proceso histórico de emancipación inacabado alberga diversas corrientes ideológicas y que, por lo tanto, la historia de los feminismos es diversa y no se reduce a la historia del feminismo liberal.

Así, puede afirmarse que la participación política de las mujeres no inicia con la lucha por el sufragio ni navega necesariamente por las olas del feminismo relata- 
das tradicionalmente, ni en Colombia ni en otras sociedades que hayan atravesado procesos de industrialización, donde fueron las mujeres, niñas y niños la fuerza de trabajo más explotada. De esta manera, resalta la vinculación de las mujeres con el trabajo remunerado como un aspecto fundamental en las condiciones de posibilidad para la generación de procesos de resistencia que no han sido plenamente documentados. Dado lo anterior, puede afirmarse que el feminismo popular es anterior a los movimientos sufragistas, y se encuentra en luchas concretas de algunos grupos poblacionales subalternos en espacios considerados públicos y privados.

Para el caso colombiano, el feminismo popular de las primeras décadas del siglo xx se caracterizó por estar construido por las mujeres trabajadoras del campo y la ciudad, que no sólo participaron como mujeres en luchas sociales, sino que lo hicieron con perspectiva feminista, abordando temas como el machismo, la sexualidad, la igualdad salarial, la reducción de la jornada laboral y las violencias relacionadas con el género. Estas mujeres transgredieron estereotipos y roles hegemónicos asignados a la feminidad, al manifestarse políticamente en espacios públicos que no estaban pensados para ellas; además, se vincularon masivamente a la industria cuando se su espacio asignado era el doméstico - no siempre por elección sino por necesidad-, soportando estigmatización y maltrato por ser mujeres trabajadoras.

En el marco de este feminismo se utilizaron como repertorios de acción las publicaciones independientes (como la revista Feminismo de 1919), u otras publicaciones en periódicos obreros, así como los centros de estudio para educar de manera crítica a las mujeres empobrecidas excluidas de las instituciones formales. Fueron representativos del feminismo popular repertorios como las huelgas o paros laborales, como formas de presión para la consecución de sus peticiones. Respecto a lo organizativo, en este feminismo resalta la generación de organizaciones o iniciativas de mujeres articuladas a otras de mayor alcance poblacional o temático como las socialistas, lo cual expresa el reconocimiento de la importancia de la articulación de las luchas, en contraste con tendencias separatistas del feminismo o del socialismo.

Por otro lado, se identifica que la relación entre feminismo y socialismo se estableció, por un lado, a través de las publicaciones realizadas por periódicos obreros y socialistas, que promovieron el debate de estas ideas y difundieron iniciativas de luchas feministas y socialistas. Por otro lado, se dio la articulación de luchas contra las contradicciones basadas en la clase socioeconómica, la etnia y el género, demostrando relaciones de carácter orgánico, así como programáticas y estratégicas, en algunas reivindicaciones del Partido Socialista, de las organizaciones de Montería y la huelga en Bello. Otra forma de relacionamiento fue a partir del apoyo planificado de luchas concretas desde Partido Socialista y posteriormente el PSR a organizaciones y movilizaciones de mujeres.

Como puede notarse, hubo importantes avances en el establecimiento de esta relación. El Partido Socialista y el PSR reconocieron la situación laboral de las mujeres y acompañaron las luchas por el mejoramiento de sus condiciones de trabajo, lo cual implicó debates sobre las violencias de género y la justicia para las mujeres. A su vez, reconocieron otros temas como la capacidad de administrar sus bienes de manera independiente sobre el esposo. Pese a esto, se presentaron retos para territorializar planteamientos programáticos de este tipo, que implicaban cambios en la 
concepción sobre las mujeres. Esto se evidencia en los discursos machistas y misóginos publicados en algunos de sus órganos de difusión, como los expuestos en $\mathrm{El}$ Luchador, así como en la práctica de construcción de liderazgos, que fue principalmente masculina aunque existieran muchas mujeres reconocidas trabajando en el partido, como en el caso del PSR.

Por otro lado, se da una evidente acogida de las ideas socialistas en las organizaciones de Montería y en la huelga de la Fábrica de Tejidos de Bello en 1920. Allí sus reivindicaciones lograron estar mejor integradas y los liderazgos más fuertes fueron los femeninos, aunque con participación masculina. Dado esto puede afirmarse que se presentó una mejor acogida del socialismo en las mujeres que del feminismo en las organizaciones socialistas dirigidas por hombres.

Esta relación no se estableció de manera homogénea debido a que algunas de las experiencias analizadas se desarrollaron en tiempos y territorios diferentes, lo que las enmarca en contextos diversos. Es así que podría decirse que en la costa norte hubo mayor favorabilidad para la acogida y articulación de las ideas feministas y socialistas por su posicionamiento geográfico, que permitió la entrada de personas e ideas al país, en contraste con la situación de Antioquia para las primeras décadas del siglo xx, que estuvo marcada por el conservadurismo y el control de la Iglesia, lo cual pudo influir en la manera en cómo se desarrollaron y nombraron las luchas. De igual manera, la relación entre feminismo y socialismo no fue lineal, presentó un retroceso a partir de 1930, no sólo influido por el Partido Comunista, sino también por la llegada del Partido Liberal al Gobierno Nacional y la salida de las mujeres de la industria fabril.

Las experiencias estudiadas, su forma de relacionamiento, transformaciones y contextos; aportan a la comprensión histórica de los procesos políticos. Permiten identificar cómo se configuraron, mantuvieron y transformaron relaciones de poder entre diferentes discursos, tales como el feminismo y el socialismo.

Actualmente esta relación sigue siendo problémica; por ello, con el presente trabajo se buscó aportar a la identificación de debates, avances y retrocesos históricos para contribuir a la superación del actual estancamiento del debate en varios sectores de la izquierda en Colombia. Pese a esto cabe recordar que lo importante es no dejar de caminar, pues, como afirmó Juana Julia Guzmán, «el cobarde no hace historia». 


\section{REFERENCIAS BIBLIOGRÁFICAS}

Acosta Restrepo, Alfonso. «Intervención de Don Alfonso Acosta Restrepo», en Cano Márquez, María, María Cano y su época. Flor del trabajo y semilla de esperanza, memorias de su centenario. Medellín: Litoarte, 1988, pp. 69-80.

Álvarez, Ana. «La articulación del feminismo y el socialismo en el siglo xıx: el conflicto clase género». Recuperado de http://acoca2.blogs.uv.es/files/2013/11/7-Feminismo-y-socialismo.pdf.

Aponte, Elida. «El feminismo y el socialismo: encuentros y desencuentros. La propuesta en el marco de la Revolución Bolivariana de Venezuela», en Frónesis, vol. 21, n. ${ }^{\circ}$ 1, (2014), pp. 136-164.

Archila, Mauricio. «Intimidad y sociabilidad de los sectores obreros durante la primera mitad del siglo xx», en Historia de la vida privada en Colombia. Bogotá: Taurus, (2011), pp. 151-179.

Atehortúa, Juan (1918, octubre 10). Por las obreras. El luchador.

BARba, Sandra. «Romper las olas de la historia feminista», en Letras Libres, (2017). Recuperado de http://www.letraslibres.com/mexico/historia/romper-las-olas-la-historia-feminista.

Bebel, Augusto. La mujer en el pasado, en el presente, en el porvenir. Madrid: F. Granada y Ca, 1906.

Bonilla, Gloria. «La lucha de las mujeres en América Latina: Feminismo, ciudadanía y derechos», en Palabra, n. ${ }^{\circ} 8,2007$, pp. 42-59.

Brenner, Johanna. «El feminismo socialista en el siglo XXI» en Sin permiso, pp. 1-9, 2014. Recuperado de http://www.sinpermiso.info/sites/default/files/textos/brenn.pdf.

CARo, Edgar. Marx, marxistas y socialistas en Colombia 1919-1930. Universidad Nacional de Colombia. Bogotá: Facultad de Ciencias Humanas, departamento de Historia, 2017.

Castañeda, Martha. Metodología de la investigación feminista. México: Fundación Guatemala. Centro de Investigaciones Interdisciplinarias en Ciencias y Humanidades. Universidad Nacional Autónoma de México, 2008.

Chávez, Hugo. El socialismo del siglo XXI. Venezuela: Ministerio del Poder Popular para la Comunicación y la Información. Colección Cuadernos para el Debate, 2011. Recuperado de http://www.minci.gob.ve/wp-content/uploads/downloads/2013/01/reflexiones_del_siglo_ xxicdw.pdf.

Cohen, Lucy. Colombianas en la vanguardia. Colección Clío. Medellín: Editorial Universidad de Antioquia, 2001.

Colmenares, Ana. "Investigación-acción participativa: una metodología integradora del conocimiento y la acción». Voces y Silencios: Revista Latinoamericana de Educación, vol. 3, n. ${ }^{\circ}$ 1, (2002), pp. 102-115.

Confederación Obrera de Bogotá. (1919, Julio 01). Plataforma socialista. El Luchador.

Correa, Hernán. «La cotidianidad cultural y política en los años veintes», en CANo Márquez, María, María Cano y su época. Flor del trabajo y semilla de esperanza, memorias de su centenario. Medellín: Litoarte, 1988, pp. 10-23.

¿CuÁndo es más fea la mujer? (1918, septiembre 26). El luchador.

Detective (1920, febrero 17). Entrevista con la Señorita Betsabé Espinal. El Luchador.

Directorio Socialista Nacional (1920, marzo 7). Habla el directorio socialista nacional. El Luchador. 
ElJach, Matilde. «El baúl de Juana Julia "el cobarde no hace historia”». Revista CEPA, año viII, volumen II, número 17, (2013). Recuperado de https://revistacepa.weebly.com/ uploads/1/3/3/7/13372958/el_cobarde_no_hace_historia.pdf.

ENGEls, Frederich. Del socialismo utópico al socialismo cientifico, (1876). Recuperado de https://www. marxists.org/espanol/m-e/1880s/dsusc/1.htm\#_ftnref18.

Fabbri, Luciano. Apuntes sobre Feminismos y construcción de Poder Popular. Rosario: Puño y Letra Editorial ismo de Base, 2013.

Fals, Orlando. Historia doble de la costa, volumen 4. Colombia: Facultad de Derecho, Ciencias Políticas y Sociales de la Universidad Nacional de Colombia, 2002.

FALs, Orlando. El socialismo raizal y la Gran Colombia bolivariana. Caracas: Investigación Acción Participativa. Caracas, Venezuela, 2008.

Federici, Silvia. El patriarcado del salario, criticas feministas al marxismo. Madrid: Traficantes de sueños, 2018.

FLórez, Carlos. «Identidades políticas del socialismo en Colombia 1920-1925. Opinión Jurídica», vol. 9, (2010), pp. 167-191.

García, Álvaro. Socialismo comunitario un horizonte de época. Bolivia: Vicepresidencia del Estado, Presidencia de la Asamblea Legislativa Plurinacional, 2015.

Guaramato, Liz. Tierra mujeres y hombres libres, relaciones de género en el Frente Nacional Campesino Ezequiel Zamora. Venezuela: Editorial La Estrella Roja, 2015.

Hall, Stuart. "Notas sobre la desconstrucción de "lo popular"», en Samuel, Ralph (ed.), Historia populary teoría socialista. Recuperado de http://www.ram-wan.net/restrepo/hall/notas\%20 sobre $\% 201 \mathrm{l} \% 20$ deconstruccion $\% 20 \mathrm{de} \% 20 \mathrm{lo} \% 20$ popular.pdf.

Hartmann, Heidi. «The Unhappy Marriage of Marxism and Feminism: Towards a More Progressive Union», en Marx Today. New York: Palgrave Macmillan, 2010, pp. 201-228.

Herrera, Eloy (1920, marzo 7). ¡Aleluya! El luchador.

Instituto de Cultura Femenina (junio de 1929). Letras y Encajes, vol. 35. p. 569.

Korol, Claudia. Socialismo y feminismo en el horizonte de nuestras luchas populares, 2013. Recuperado de https://viacampesina.org/es/wp-content/uploads/sites/3/2013/05/ES-07.pdf.

La Desgracia (1919, mayo 20). El Luchador.

La Dirección (agosto de 1929). Letras y Encajes. Letras y Encajes, vol. 1. p. 2.

La huelga de Bello (1920, marzo 9). El Luchador.

Lugones, María. Colonialidad y género. USA: Binghamton University, 2008.

Luis, Carlos (1920, febrero 20). Labor del socialismo. El Luchador.

Luna, Lola y FAgOAga, Concha. «Notas para una historia social del movimiento de las mujeres: signos radicales y signos reformistas", en Ordenamiento jurídico y realidad social de las Mujeres. Madrid: UAM, 1985. Recuperado de http://www.lolagluna.com/publicaciones.html.

Luna, Lola. El sujeto sufragista feminismo y feminidad en Colombia, 1930-1957. Cali: Centro de Estudios de Género, Universidad del Valle/La Manzana de la Discordia, 2004.

Martínez, Yaneth \& Uribe, Carlos. Betsabé Espinal, la natural: itinerario de una lucha. Bello, Colombia, 1920. Fondo Editorial Quitasol, 2013.

Mitchell, Juliet. Women's Estate. New York: Vintage Books, 1973. 
Nuñez, Luz. El obrero ilustrado prensa obrera y popular en Colombia 1909-1929. Andes: Universidad de los Andes, Facultad de Ciencias Sociales-CESO, Departamento de Historia, 2006.

Olive, Aleira (junio de 1929). Doña Carolina Lena etc. Letras y Encajes, vol. 35.

Protesta de las huelguistas de Bello (1920, marzo 16). El Correo Liberal.

Reyes, Catalina. Aspectos de la vida social y cotidiana en Medellin, 1890-1930. Bogotá: Colcultura, 1996.

Sandoval, Chela. Methodology of the oppressed. Minnesota: The University of Minnesota, 2000.

SoluCión de la huelga (1920, marzo 7). El Luchador.

SuÁrez, Gina. La «Confluencia de Mujeres» en el «Congreso de los Pueblos»: nuevos escenarios y viejos debates para el movimiento de mujeres. Bogotá: Universidad Nacional de Colombia, 2016.

Thompson, Becky. «Multiracial feminism: recasting the chronology of second wave feminism». Feminist Studies, vol. 28, Issue 2, 2002, pp. 336-360.

Tintorero. (1920, febrero 27). Lo del lunes en Bello. El Luchador.

Toвón, Gloria. «Intervención de Gloria Tobón», en CANo Márquez, María, María Cano y su época. Flor del trabajo y semilla de esperanza, memorias de su centenario. Medellín: Litoarte, 1988, pp. 103-106.

Triunfo del socialismo en Antioquia (1920, marzo 9). El Luchador.

UnA obrera (1919, julio 23). La mujer y el Socialismo. El Luchador.

URIBE, Benedicto (1920, febrero 14). Telegrama. El Luchador.

URIBE, Carlos. Los años veinte en Colombia: ideología y cultura. Bogotá: Ediciones Aurora, 1985.

URIBE, Maria. Los años escondidos sueños y rebeldías en la década del veinte. Bogotá: Opciones Gráficas Editores, 2015.

Uribe, Ricardo (1914). Notas feministas. Tesis presentada para optar al título de doctor en Derecho y Ciencias Políticas. Universidad de Antioquia, 1914.

VALDÉs, Gilberto. «Planeta Tierra: movimientos antisistémicos». Primer Coloquio Internacional In Memoriam Andrés Aubry, 2015. Recuperado de http://seminarioscideci.org/ColoquioAndresAubry/PONENCIAS2.pdf.

VAllés, Miguel. Técnicas cualitativas de investigación social. Reflexión metodológica y práctica profesional. Madrid: Editorial Síntesis, 1999.

Valverde, Octavio. «Intervención de Octavio Valverde», en Cano Márquez, María, María Cano y su época. Flor del trabajo y semilla de esperanza, memorias de su centenario. Medellín: Litoarte, 1988, pp. 62-66.

VeGA, Renán. Gente muy rebelde 3. Mujeres, artesanos y protestas cívicas. Bogotá: Ediciones Pensamiento Crítico, 2002.

Villa, María. «Intervención de la pintora María Villa», en Cano Márquez, María, María Cano y su época. Flor del trabajo y semilla de esperanza, memorias de su centenario. Medellín: Litoarte, 1988, p. 62.

Villareal, Norma. Movimientos de mujeres y participación politica en Colombia 1930 -1991. Barcelona: Seminario interdisciplinar Mujeres y Sociedad, 1994.

VIVEros, Mara. La interseccionalidad: una aproximación situada a la dominación. Bogotá: Universidad Nacional de Colombia, 2016. 
Zapata, Arístides (1920, febrero 20). Las obreras de Bello. El Luchador.

Zapata, Arístides (1919, junio 10). Semanalmente. El Luchador. 